\title{
The Spatially Resolved H $\alpha$-Emitting Wind Structure of P Cygni
}

\author{
Aurelian Balan ${ }^{1,2}$, C. Tycner ${ }^{1}$, R. T. Zavala ${ }^{3}$, J. A. Benson ${ }^{3}$, D. J. Hutter ${ }^{3}$, M. Templeton ${ }^{4}$
}

\begin{abstract}
High spatial resolution observations of the $\mathrm{H} \alpha$-emitting wind structure associated with the Luminous Blue Variable star P Cygni were obtained with the Navy Prototype Optical Interferometer (NPOI). These observations represent the most comprehensive interferometric data set on $\mathrm{P}$ Cyg to date. We demonstrate how the apparent size of the $\mathrm{H} \alpha$-emitting region of the wind structure of $\mathrm{P}$ Cyg compares between the 2005, 2007 and 2008 observing seasons and how this relates to the $\mathrm{H} \alpha$ line spectroscopy. Using the data sets from 2005, 2007 and 2008 observing seasons, we fit a circularly symmetric Gaussian model to the interferometric signature from the $\mathrm{H} \alpha$-emitting wind structure of $\mathrm{P}$ Cyg. Based on our results we conclude that the radial extent of the $\mathrm{H} \alpha$-emitting wind structure around $\mathrm{P}$ Cyg is stable at the $10 \%$ level. We also show how the radial distribution of the $\mathrm{H} \alpha$ flux from the wind structure deviates from a Gaussian shape, whereas a two-component Gaussian model is sufficient to fully describe the $\mathrm{H} \alpha$-emitting region around $\mathrm{P}$ Cyg.
\end{abstract}

Subject headings: stars: winds, outflows - stars: individual (P Cyg) - techniques: interferometric

\section{Introduction}

Luminous Blue Variable (LBV) star P Cyg (HD 193237, B2pe) is an unusual star with a unique stellar wind structure. LBVs are evolved, very luminous, massive supergiant stars

\footnotetext{
${ }^{1}$ Department of Physics, Central Michigan University, Mount Pleasant, MI 48859; abalan@delta.edu, c.tycner@cmich.edu.

${ }^{2}$ Current address: Delta College, Science Division, University Center, MI, 48710

${ }^{3}$ US Naval Observatory, Flagstaff Station, 10391 W. Naval Observatory Rd., Flagstaff, AZ 86001; bzavala@nofs.navy.mil, jbenson@nofs.navy.mil, djh@nofs.navy.mil

${ }^{4}$ American Association of Variable Star Observers, 49 Bay State Road, Cambridge, MA 02138; matthewt@aavso.org
} 
that show some type of instability. As the likely progenitors of Wolf-Rayet stars (Crowther 2007) LBV's may provide insight for the ultimate end-stage of these stars, the core-collapse supernovae. P Cyg was discovered in 1600 after a violent mass-loss event caused it to quickly brighten to a third magnitude star (de Groot 1969). The violent mass-loss events that spur these eruptions are not fully understood, but a number of competing models including single and binary star scenarios attempt to explain these eruptions (Humphrevs \& Davidson 1994). With a mass-loss between $2 \times 10^{-5}$ and $4 \times 10^{-4} M_{\odot} \mathrm{yr}^{-1}$ (van Blerkom 1978; Abbott et al. 1980; Leitherer \& Zickgraf 1987), P Cyg displays characteristics that are suggestive of energetic mass outflows. It is for this reason that $\mathrm{P}$ Cyg has a unique stellar wind structure with possible high density regions in motion within the wind structure. Despite numerous studies published in the literature that discuss P Cyg or other LBVs, there is still need for observations that can spatially resolve the circumstellar region around P Cyg.

The spectrum of $\mathrm{P}$ Cyg reveals emission line profiles with their archetypical shapes, which are formed by the circumstellar matter that has been shed from the central star. These profiles are commonly identified by an emission component to the red side of an absorption line. The origin of this Doppler shift can be explained with a spherically symmetric outflowing wind in which the velocity increases with radial distance (see, e.g., Lamers \& Cassinelli 1999, $§ 2.2)$. The absorption component corresponds to the continuum light absorbed by the wind structure directly between the observer and the central star. The emission component on the other hand originates in the spherically symmetric halo around the central star. Because the same characteristics apply to the $\mathrm{H} \alpha$ emission line, which is one of the strongest emission lines in the spectrum of $\mathrm{P} \mathrm{Cyg}$, this makes the $\mathrm{H} \alpha$ emission line an excellent probe of the wind region around $\mathrm{P}$ Cyg.

P Cyg currently has an estimated mass of $30 \pm 10 M_{\odot}($ Lamers et al. 1983, 1985). At the start of its lifetime P Cyg possibly had an initial mass of $50 M_{\odot}$ (de Groot \& Lamers 1992), but has shed much of its mass because of its violent history of mass loss events. The effective temperature and the stellar diameter of $\mathrm{P}$ Cyg are estimated to be $\mathrm{T}_{\text {eff }}=19,300 \pm 2000 \mathrm{~K}$ and $76 \pm 14 R_{\odot}$, respectively (Lamers et al. 1983). However, the $\mathrm{H} \alpha$-emitting region extends radially much farther than the central star allowing us to resolve this region using longbaseline interferometric techniques, even though the distance is estimated to be in the range of $1.7 \pm 0.1 \mathrm{kpc}$ to $1.8 \pm 0.1 \mathrm{kpc}$ (Lamers et al. 1983; Najarro et al. 1997).

The circumstellar region of $\mathrm{P}$ Cyg has been spatially resolved in the past using radio and optical interferometry, as well as direct imaging with adaptive optics (AO). Radio interferometric observations detect the nebula around $\mathrm{P}$ Cyg at the angular scales from $\sim 50$ mas to almost an arcminute (Skinner et al. 1997, 1998), whereas optical long-baseline interferometry is sensitive to structures in the nebula of P Cyg that are more than an or- 
der of magnitude smaller. Therefore, optical interferometry provides a unique window of opportunity to resolve the inner portion of the wind structure.

Most relevant to our study are the results that probed the $\mathrm{H} \alpha$-emitting region of $\mathrm{P}$ Cyg. For example, based on near-diffraction limited observations obtained using AO system on a $1.52 \mathrm{~m}$ telescope, Chesneau et al. (2000) not only resolved the outer H $\alpha$-emitting region of the extended envelope, but detected signatures of clumping. Although, the angular scales sampled with a $1.52 \mathrm{~m}$ telescope were quite large, in the region of 600 mas, the angular

resolution was limited to 50 mas. On much smaller angular scales, Vakili et al. (1997) using a single $17.7 \mathrm{~m}$ baseline of the GI2T interferometer have resolved the inner $\mathrm{H} \alpha$-emitting envelope of P Cyg yielding an angular size of $5.52 \pm 0.47$ mas and reported a marginal detection at the HeI 6678 line. The spatial scales probed by our study are similar to those sampled by Vakili et al. (1997), however, the interferometric observations at the $\mathrm{H} \alpha$ line obtained in our study were acquired using 11 unique interferometric baselines ranging in length from 15.8 to $79.3 \mathrm{~m}$, thus resulting in much higher angular resolution measurements.

\section{Observations}

\subsection{Interferometry}

High angular resolution long-baseline interferometric observations were obtained on P Cyg over thirty-seven nights in 2005, 2007 and 2008 using the Navy Prototype Optical Interferometer (NPOI) located near Flagstaff, Arizona. The NPOI is a long-baseline optical interferometer which consists of six $50 \mathrm{~cm}$ siderostat mirrors that send $12.5 \mathrm{~cm}$ diameter light beams down the optical feed system consisting of $15 \mathrm{~cm}$ diameter pipes to an optics lab where the interference (i.e., a fringe) between the beams is recorded (Armstrong et al. 1998). Observations covering a wide spectral band can be taken over multiple baselines (currently ranging from 19 to $80 \mathrm{~m}$ in length), and are geometrically compensated and modulated using vacuum delay-lines to guarantee the entire band is being observed simultaneously.

The observing process involves measuring the fringe contrast over 15 spectral channels every $2 \mathrm{~ms}$. Each $2 \mathrm{~ms}$ frame is then used to obtain a squared visibility $\left(V^{2}\right)$ measure (i.e., a $\mathrm{V}^{2}$ value measures the fringe contrast). Afterward, these values are incoherently averaged over $1 \mathrm{~s}$ intervals to create $1 \mathrm{~s}$ data points (Hummel et al. 2003). The final observations consist of 30 s of integration (also known as a "scan") during which the squared visibilities are measured simultaneously at three baselines per output from the beam-combiner (with two outputs being used simultaneously) and 15 spectral channels covering the wavelengths between 560 and $870 \mathrm{~nm}$. The typical observational sequence consists of a pair of coherent 
and incoherent (off the fringe) scans on a target followed by a pair of scans on a calibrator. The incoherent scans are used to estimate the additive bias terms affecting the squared visibility measures. The process is repeated during a night for as long as the fringe packet can be detected on the target star. Typically, the observations stop when the optical path difference between the different siderostats cannot be compensated anymore.

The H $\alpha$-emitting sources form a specific class of targets for NPOI since the entire emission line ends up only in one out of the 15 spectral channels. This characteristic allows one to calibrate the $V^{2}$ values from the channel containing the $\mathrm{H} \alpha$ emission using the $V^{2}$ values from the neighboring continuum channels (Tycner et al. 2003). Although during this calibration process one needs to assume that the diameter of the central star at the continuum wavelengths is known, the final results based on the $\mathrm{H} \alpha$ emission are only weakly dependent on the adopted value for the angular diameter of the central star (we explore this in more detail in $\S 3.2$ ). For the purpose of the calibration we adopted a uniform disk diameter of 0.2 mas to represent the central star at the continuum wavelengths. We obtain this diameter based on a distance estimate of $1.8 \pm 0.1 \mathrm{kpc}$ and a central star radius of $76 \pm 14 R_{\odot}$ (Lamers et al. 1983).

Applying the procedures described above to the entire observational data set we acquired for P Cyg, yielded a total of 1,534 H $\alpha$ squared visibilities on 11 unique baselines (out of a maximum of 15 unique baselines for a 6 -station configuration). Table 1 lists the number of unique $\mathrm{H} \alpha V^{2}$ measures we obtained for each of the nights in our observing campaign. Although the observational setup for each of the three observing seasons was slightly different, and therefore a range of different baselines were sampled each year, in total we have obtained observations at baselines ranging from the shortest $(15.8 \mathrm{~m})$ to the longest $(79.3 \mathrm{~m})$ possible at the time of our observations. More specifically, the 2005 and 2008 seasons contained baselines between 18.9 and $53.2 \mathrm{~m}$, and the 2007 season contained baselines from 15.8 to $79.3 \mathrm{~m}$ in length. Figure 1 shows the sampling of the $(u, v)$-plane in the $\mathrm{H} \alpha$ channel for P Cyg based on all the available nights in 2005, 2007 and 2008. The "arcs" seen in the plot are a result of the changing projection of the baseline vector on the sky during the night due to Earth's rotation. Three seasons of squared visibility data sampled across the $(u, v)$-plane are shown in Figure 2 plotted as a function of radial spatial frequency $\left(\sqrt{u^{2}+v^{2}}\right)$. The calibrated $\mathrm{H} \alpha V^{2}$ measures are listed in Table 2 .

In addition to the $V^{2}$ measures we have also obtained closure phase quantities (sum of complex phases on three baselines forming a triangle) on four unique baseline triangles. By fitting a quadratic function to the closure phases from the continuum channels (at each scan and closure phase configuration separately), and then removing this quadratic trend from all channels, including the $\mathrm{H} \alpha$ channel, we were able to obtain the differential closure phases for 
the $\mathrm{H} \alpha$-emitting region. Because we expect the central star to be mostly unresolved at the continuum channels and if we assume that the continuum source is point-symmetric, then we expect the closure phases to cluster around a zero value. This allows us to inspect the $\mathrm{H} \alpha$ closure phases with respect to the mean zero closure phase. In order to reduce the random noise present in data associated with a single scan we calculated the weighted mean closure phases for each season and each unique configuration. In the 2007 season one of the baselines (AE-AN) was sampled at two independent outputs from the beam combiner and this allowed us to calculate two closure phase quantities containing this baseline either from one output beam or the other. Because the observations for the other baselines were identical, we decided not to average these quantities, and instead treat them separately. Figure 3 shows the weighted mean closure phases for all the configurations with the quadratic trend based on the continuum channels already removed.

\subsection{Spectroscopy}

The $\mathrm{H} \alpha$ emission line, as detected by the NPOI, falls on a spectral channel that has a spectral width of $15 \mathrm{~nm}$. Therefore, the interferometric data obtained on $\mathrm{P}$ Cyg does not provide sufficient spectroscopic information to allow us to determine the strength or the shape of the $\mathrm{H} \alpha$ emission line. For this reason, we obtained complementary spectroscopy of P Cyg using the Lowell Observatory's John S. Hall $1.1 \mathrm{~m}$ telescope located at the same observing site as the NPOI. The telescope was outfitted with the Solar-Stellar Spectrograph (SSS), which is a fiber-fed echelle spectrograph (Hall \& Lockwood 1995). Spectroscopic data on P Cyg were obtained during the period from April 2005 to December 2007, with the individual nights listed in Table 3. The SSS instrument produces spectra in the $\mathrm{H} \alpha$ region with a resolving power of $\sim 10,000$. A typical $\mathrm{H} \alpha$ line profile normalized with respect to the continuum level of $\mathrm{P}$ Cyg is shown in Figure 4. The equivalent width (EW) measurements based on the individual spectra are also plotted in the upper panel of Figure 5.

\section{Analysis}

\subsection{Interferometric Results}

There are two signatures in the squared visibilities from the spectral channel containing the $\mathrm{H} \alpha$ emission line shown in Figure 2, One is from the central star and the other is from the $\mathrm{H} \alpha$-emitting circumstellar region. To model this data, we use the same two component 
model as was used by Tycner et al. (2006) of the form:

$$
V_{\text {model }}^{2}=\left[c_{\mathrm{p}} V_{\mathrm{p}}+\left(1-c_{\mathrm{p}}\right) V_{\text {env }}\right]^{2}
$$

where $V_{\mathrm{p}}$ is the visibility function representing the photosphere of the central star, $V_{\text {env }}$ is the visibility function representing the circumstellar envelope, and $c_{\mathrm{p}}$ is the fractional contribution from the stellar photosphere to the total flux in the $\mathrm{H} \alpha$ channel.

Based on the results from differential closure phases (recall $\S$ 2.1), which showed very weak (if any) variations across the $\mathrm{H} \alpha$ channel, we expect the $\mathrm{H} \alpha$-emitting structure to be sufficiently well described (at the level of angular resolution provided by our range of baselines) by a model that is both point symmetric and concentric with the central star. This means that $V_{\mathrm{p}}$ and $V_{\text {env }}$ in equation 1 can be represented by real functions (i.e., the Fourier transform of real and even function results in a real function). To model the stellar photosphere we use a circularly symmetric uniform disk brightness distribution. The normalized visibility amplitude for a uniform disk can be written as:

$$
V_{\mathrm{p}}(u, v)=2 \frac{J_{1}(\pi a s)}{\pi a s}
$$

where $J_{1}$ denotes the Bessel function of the first kind and first order, $a$ is the angular diameter, and $s$ is the radial spatial frequency (i.e., $s=\sqrt{u^{2}+v^{2}}$ ). Because the interferometric observations of $\mathrm{P}$ Cyg were obtained at sufficiently high spatial frequencies so that the $\mathrm{H} \alpha$ emitting region was fully resolved, we can compare different models of brightness distribution for the envelope component. We choose to model our data with a uniform disk (UD) and a Gaussian distribution (GD), where in the former $V_{\text {env }}$ has the same functional form as equation 2 except that $a$ is replaced with $\theta_{\mathrm{UD}}$, and in the latter case

$$
V_{\mathrm{env}}^{\mathrm{GD}}=\exp \left[-\frac{\left(\pi \theta_{\mathrm{GD}} s\right)^{2}}{4 \ln 2}\right]
$$

where $\theta_{\mathrm{GD}}$ corresponds to the full-width at half-maximum (FWHM) of the Gaussian model.

The envelope component $\left(V_{\text {env }}\right)$ in equation 1, represented by either a uniform disk or a Gaussian radial distribution, was assumed to be circularly symmetric. This choice was based on a test performed on the observational data set being divided into 18 subsets based on the positional angles covered by the observations. Figure 6 shows the variation of the fitted diameter of a Gaussian model as a function of PA. Although a hint of variation is present in the data, more than $68 \%$ (based on $\pm 1 \sigma$ expectation) of points fall within $5.5 \%$ of the mean value and thus we conclude that some of that variation might be solely due to the fact that the observations were acquired over many observing seasons and intrinsic variability at the $5 \%$ level cannot be ruled out. Similar test performed on much smaller data set from only 
one season does not reveal any variation beyond those expected based on the uncertainties associated with the fitted model parameters. Therefore, for the purpose of describing the geometrical structure of the $\mathrm{H} \alpha$-emitting region of $\mathrm{P}$ Cyg, we decided to concentrate only on circularly symmetric models fitted to the $V^{2}$ data from the individual observing seasons, as well as to the entire data set.

Table 4 summarizes both UD and GD model results along with their corresponding reduced $\chi^{2}$ values $\left(\chi_{\nu}^{2}\right)$, which were used to assess the goodness-of-fit of each model. Based on the $\chi_{\nu}^{2}$ values obtained from model fits to the entire data set, it is clearly evident that the Gaussian model (with $\chi_{\nu}^{2}=1.9$ ) produced a significantly better fit to the data than a circularly symmetric uniform disk model (with $\chi_{\nu}^{2}=2.8$ ). However, neither the UD nor GD model fully reproduces the trend seen in the data obtained at high spatial frequencies as can be seen in Figure 2. Although, the Gaussian model does produce a better fit, to fully account for the observational signature at the high spatial frequencies we require a model with an extra degree(s) of freedom. A natural extension to a Gaussian model is a two-component Gaussian model of the form

$$
V_{\mathrm{env}}^{2 \mathrm{GD}}=\mathrm{k}_{1} \exp \left[-\frac{\left(\pi \theta_{1} \mathrm{~s}\right)^{2}}{4 \ln 2}\right]+\left(1-\mathrm{k}_{1}\right) \exp \left[-\frac{\left(\pi \theta_{2} \mathrm{~s}\right)^{2}}{4 \ln 2}\right],
$$

where $\theta_{1}$ and $\theta_{2}$ correspond to the FWHM values of the two Gaussian components, and $\mathrm{k}_{1}$ is the fractional contribution from the first Gaussian. Similar two-component Gaussian fits have been applied to other stars by Hofmann et al. (2002) and Preibisch et al. (2003). Combining equations 1, 2 and 4, and fitting the two-component Gaussian model to the observations from all three observing seasons results in angular diameters of $\theta_{1}=5.64 \pm 0.17$ mas and $\theta_{2}=$ $1.80 \pm 0.11$ mas, with a fractional contribution from the stellar photosphere, $\mathrm{c}_{\mathrm{p}}=0.70 \pm 0.01$ and $\mathrm{k}_{1}=0.57 \pm 0.02$ (i.e., with the two Gaussian components contributing approximately equally to the net $\mathrm{H} \alpha$ emission). The best-fit two-component Gaussian model had the lowest reduced $\chi^{2}$ value (yielding $\chi_{\nu}^{2}$ of 1.5 based on the entire data set) out of all the models considered in our analysis. The model curve corresponding to the two-component Gaussian model is also shown in Figure 2,

The success of the two-component Gaussian model to reproduce the observational signature from the $\mathrm{H} \alpha$ channel is mostly due to the higher degree of freedom along the radial direction. In other words, one can in principle approximate any monotonically decreasing function as a sum of a large number of Gaussian functions. In fact, any other monotonically decreasing function can also be used instead of a Gaussian. For example, a sum of a Gaussian distribution and a uniform disk component of the form

$$
V_{\mathrm{env}}^{\mathrm{UD}+\mathrm{GD}}=l_{1} 2 \frac{J_{1}\left(\pi \theta_{\mathrm{UD}} s\right)}{\pi \theta_{\mathrm{UD}} s}+\left(1-l_{1}\right) \exp \left[-\frac{\left(\pi \theta_{\mathrm{GD}} s\right)^{2}}{4 \ln 2}\right],
$$


where $\theta_{\mathrm{UD}}$ and $\theta_{\mathrm{GD}}$ correspond to the angular diameters of the uniform disk and Gaussian components, respectively, and $l_{1}$ is the fractional contribution from the uniform disk, yields equally good fit to the data as the two-component Gaussian model (resulting in a reduced $\chi^{2}$ of 1.5). The best-fit angular diameters are in this case: $\theta_{\mathrm{UD}}=3.06 \pm 0.15$ mas and $\theta_{\mathrm{GD}}=5.46 \pm 0.16$ mas, with a fractional contribution from the stellar photosphere, $c_{\mathrm{p}}$, of 0.72 and $l_{1}=0.36$.

\subsection{Central Star Diameter}

A central star diameter of 0.2 mas was assumed for our model (recall $\S$ 2.1). Because varying the adopted diameter for the central star affects the calculated size of the $\mathrm{H} \alpha$ emitting region, we test the dependency of the angular diameter of the $\mathrm{H} \alpha$-emitting wind structure surrounding $\mathrm{P}$ Cyg on the angular diameter of the photospheric component. This is done by fitting equation 1 to the observations with the envelope component represented by a Gaussian model and the photospheric component with a UD model for a range of adopted stellar diameters. Using the observations from the 2007 observing season we find that the assumed central star diameter of P Cyg at most has only a $1 \%$ effect on the derived angular size of the wind structure when the adopted angular diameter of the central star is varied from 0 to 1 mas. Therefore, an angular size of the continuum-emitting region that is underestimated by a factor of five results only in a $1 \%$ effect on our best-fit angular diameter of the $\mathrm{H} \alpha$-emitting region.

\subsection{Spectroscopic Results}

To test for any possible link between the changes in the strength of the $\mathrm{H} \alpha$ emission line and any variations in the size of the $\mathrm{H} \alpha$-emitting region we examined the spectroscopic data for any variability on a time-scale comparable to our interferometric observations. We found that there was EW variability with an amplitude $\lesssim 10 \AA$ over a time scale of $\sim 2$ months, which is consistent with the short-term variability seen by Markova et al. (2001b). However, the yearly mean $\mathrm{H} \alpha \mathrm{EW}$ and yearly mean $\mathrm{H} \alpha$ peak signal showed no variation from year to

year during our observing period suggesting relative stability in EW measure on a year-toyear time scale (see Table 3).

While our mean spectroscopic data suggest stability on a year-to-year scale, we cannot rule out variability of $\mathrm{H} \alpha$ emission if such changes are correlated with changes in the continuum level as suggested by Markova et al. (2001a). If the continuum brightened and the 
$\mathrm{H} \alpha$ has increased in strength by the same fractional amount, it would appear stable. Regardless of this apparent mean stability, we detect high frequency variations on time scales much shorter than one year. The upper panel in Figure 5 shows the H $\alpha$ equivalent widths derived from our spectroscopic observations along with the data published by Markova et al. $(2001 a)$.

Markova et al. (2001b) obtained both spectroscopic and photometric observations of P Cyg and found that the EW measurements displayed a slow variability, with an amplitude of about $30 \AA$ and a duration of about 600 days, and a faster variability with an amplitude up to $10 \AA$ and duration of 40 to 60 days. While we were able to observe a variability of up to $10 \AA$ in amplitude on a short (month-to-month) time scale, we did not observe the long-term $30 \AA$ amplitude variability. If the long-term variability discussed in Markova et al. (2001b) was present at the time of their observations, it is no longer present in our data. Therefore, based on our spectroscopic and interferometric observations, we conclude that $\mathrm{P}$ Cyg is in a quiescent phase of its lifetime, and it is not going through any drastic changes.

The variability of the $\mathrm{H} \alpha \mathrm{EW}$ in our data set is similar to the $10 \AA$ variation seen in the literature on a time scale of $\sim 2$ months. Based on our analysis of the equivalent width measures listed in Table 3, we conclude that the root mean square (RMS) variations are less than $10 \%$, and typical closer to the 2-5\% range. Therefore, for the purpose of our study we assume that the overall emission in the $\mathrm{H} \alpha$ line throughout our interferometric run was stable at the $10 \%$ level. We also tested for variability in the $\mathrm{H} \alpha$ strength during a single night and found it to be virtually non-variable as well (see Table 5). The variations were within the expected observational uncertainties of $\sim 3 \%$, mostly due to uncertainties associated with continuum level normalization.

\section{Discussion}

\subsection{The Radial Intensity Falloff}

The deviation from a Gaussian shape of the spatially resolved wind structure of P Cyg is one of our most intriguing results. Examining the squared visibilities in Figure 2, it is apparent that the observations at high spatial frequencies cannot be represented by a single-component Gaussian model. It is worthwhile to mention that the Gaussian intensity distribution is still a closer approximation to the radial intensity distribution from the wind structure than the uniform disk model (shown with dotted line in Fig. 2). To our knowledge, this is the first time the $\mathrm{H} \alpha$-emitting region of $\mathrm{P}$ Cyg have been observed to clearly deviate from a Gaussian shape. 
To reproduce our data at the highest spatial frequencies, we use the two-component Gaussian model as a way of parameterizing the spatially resolved signature of the $\mathrm{H} \alpha$ emitting region of $\mathrm{P}$ Cyg. This suggests that the wind structure is indeed more complex in P Cyg as compared to Be stars. Because P Cyg possess radiatively driven stellar wind, the velocity structure can be described by the $\beta$-law of Castor \& Lamers (1979), which implies that the wind is accelerated to its terminal velocity close to the star and then reaches a constant terminal velocity. This in turn means that the density structure of $\mathrm{P}$ Cyg will change at two different rates, one when the wind is still accelerating and one when the wind has already reached its terminal velocity at which point the density would be expected to drop with radial distance as $r^{-2}$. Although this cannot be used as a direct explanation for the two component structure because the $\mathrm{H} \alpha$ emission in $\mathrm{P}$ Cyg is optically thick (Leitherer 1988), by combining the effects of the density distribution with the radiative transfer effects it should be possible to use the data presented in this study to directly constrain a numerical wind model based on a $\beta$ velocity law. Lastly, if the two-component wind structure is dominated by optically thick and thin effects, one could argue that the the inner (optically thick) region might be better represented by a uniform disk model and the outer region by a Gaussian model. This would be in agreement with the results presented in $\$ 3.1$ that showed that the observations can be equally well represented by a two-component Uniform-Disk and Gaussian model.

P Cyg has been spatially resolved in the past using interferometric techniques. Vakili et al. (1997), using a single $17.7 \mathrm{~m}$ interferometric baseline, resolved the extended envelope of $\mathrm{P}$ Cyg at the $\mathrm{H} \alpha$ emission line. Although their data set was limited and did not allow them to fit a model more complex than a uniform disk model, the angular diameter of a uniform disk model they obtained was $\theta_{\mathrm{UD}}=5.52 \pm 0.47$ mas. They also obtained complementary $\mathrm{H} \alpha$ spectrum on $\mathrm{P} \mathrm{Cyg}$ on the same night they obtained interferometric observations with a $\mathrm{H} \alpha$ peak signal of $14.6 F_{\max } / F_{\text {cont }}$. This is somewhat weaker than the $\approx 20 F_{\max } / F_{\text {cont }}$ values we obtain based on our observations from 2005-2007 (recall Fig. 44), although the effect of lower spectral resolution in the spectra of Vakili et al. (1997) cannot be ruled out, which could explain the lower peak-to-continuum ratio seen in the observation reported by Vakili et al. (1997).

Although a uniform disk model is completely inconsistent with our observations (recall Fig. 2), in order to compare our results to Vakili et al. (1997) at effectively the same limiting spatial resolution, we fitted a uniform disk model to data from only shortest baselines (up to $18.9 \mathrm{~m}$ ). The best-fit uniform disk angular diameters ranged from 8.4 to 10.2 mas depending on which season was used in the fit (see Table 4). Therefore, our UD diameter for the $\mathrm{H} \alpha$-emitting wind structure is not consistent with the reported diameter of 5.52 mas by Vakili et al. (1997). This discrepancy might be due to the possibility that Vakili et al. (1997) 
has modeled the observational signal that contained both the mostly unresolved central star and $\mathrm{H} \alpha$-emitting envelope with only one uniform disk diameter. This would be equivalent to our equation 1, but with the $c_{\mathrm{p}}$ set to 0 , which would tend to underestimate the angular extent of the $\mathrm{H} \alpha$ emitting region. The other possibility is that the $\mathrm{H} \alpha$-emitting region actually grew in size from 1994 to 2007. If we assume that the difference between our values and those reported by Vakili et al. (1997) to be representative of a real change, then this results in an expansion rate of the $\mathrm{H} \alpha$-emitting region (due to changes in opacity) over this thirteen year period of $\sim 3 \mathrm{~km} \mathrm{~s}^{-1}$. This is well within the expected wind velocities of stars with radiation driven wind structures, especially $\mathrm{P}$ Cyg, which has a terminal wind velocity almost two magnitudes larger (Barlow et al. 1994; Lamers et al. 1985). Furthermore, if the $\mathrm{H} \alpha$-emitting region did indeed grow in size, we would expect the $\mathrm{H} \alpha$ emission to be stronger, which is supported by the fact that the $F_{\max } / F_{\mathrm{c}}$ values in Table 3 that range between 17 and 24 are larger than the $\approx 15$ peak value of the spectrum taken by Vakili et al. (1997).

\subsection{Signature of Asymmetry}

To test for the presence of a signature of deviation from point-symmetry in the signal in the $\mathrm{H} \alpha$ channel, we have plotted the weighted mean closure phases in Figure 3 . If the $\mathrm{H} \alpha$ emission originates from an intensity distribution that is not symmetric across the origin of the photocenter, or equivalently the $\mathrm{H} \alpha$ emitting region is not concentric with the central star, we would expect to see a non-zero closure phase in the $\mathrm{H} \alpha$ channel. At a close inspection of Figure 3 we conclude that the $\mathrm{H} \alpha$ closure phases are generally very close to $0^{\circ}$. Even in the cases where the mean closure phase deviates from zero values, it is only at the level of a couple of degrees, which also happens to be only 2 to 3 times the uncertainty of the mean value. Therefore, we conclude that based on our observations we do not have a strong signature of deviation from point symmetry of the $\mathrm{H} \alpha$-emitting region.

Our conclusions are not necessarily inconsistent with the a very similar detection of a subtle phase variation (at the level of $\sim 30^{\circ}$ ) across the $\mathrm{H} \alpha$ emission line detected by Vakili et al. (1997) who interpreted that as being produced by a localized spatial feature (i.e., a localized blob) within the structure of the wind. Assuming that the closure phase variations we detect across the $\mathrm{H} \alpha$ spectral channel in Figure 3 are caused by similar spatial feature (which is not necessarily expected since the observations were acquired at different epochs), we would indeed expect to see a much weaker signal across the $\mathrm{H} \alpha$ channel for two reasons. The first being that we measure the sums of three phases, which already have the tendency to lower the signal when negative and positive phases in a triangle are added together. The second effect is related to our much wider spectral channel that contains the entire $\mathrm{H} \alpha$ 
emission line and a significant contribution from the central star, which most likely can be described well by a point-symmetric intensity distribution that contains only real components in the Fourier space (i.e., only real phases). The net result is that the real components will tend to lower the net complex phase detected in the $\mathrm{H} \alpha$ channel.

Signatures of clumping in the circumstellar region of P Cyg on much larger spatial scales have also been reported. For example, based on near-diffraction limited observations using AO system on a $1.52 \mathrm{~m}$ telescope, Chesneau et al. (2000) reported clumping at the scales of 200-600 mas. Similarly, P Cyg was also imaged using the Multi-Element Radio Linked Interferometer Network (MERLIN) array by Skinner et al. (1997). Their $6 \mathrm{~cm}$ observations produced images of the circumstellar structure on scales of 100-200 mas with a 50 mas resolution, revealing structural changes on a 40 day timescale along with flux variations at the 20\% level. Skinner et al. (1997) argued that the structural variations could not be attributed to variations in the mass-loss rate. They suggested recombination within the freefree emitting region responsible for the radio emission could explain their observations. The weak signature of the non-zero closure phase in the NPOI data, if confirmed, could indicate that the clumpiness observed by Chesneau et al. (2000) and Skinner et al. (1997) already originates on scales of less than 10 mas (less than 25 stellar radii).

\subsection{Photometric Variability}

Lastly, to assess the level of photometric variability during the time frame covered by our interferometric observations, we used photoelectric V-band observations from the American Association of Variable Star Observers. The $V$-band light curve from 2005 to 2009 is shown in Figure 5 where we only include data for which the measured check star (HD 193369) magnitude was no more than 0.04 mag away from the mean value of 5.573 mag. We see both a mean seasonal-timescale variability on the order of $\sim 0.02 \mathrm{mag}$, and very short term variability (for observations taken within days to weeks) at the level of $\pm 0.05 \mathrm{mag}$. Although the short-term variability appears to have large scatter, the photometric quality of the AAVSO PEP data is generally good, with internal errors on the order of 5-10 millimagnitudes. Therefore, based on the photometric data we conclude that although the mean photometric level appears to change by less than $0.02 \mathrm{mag}$, short term variations upwards of 0.1 mag cannot be ruled out. This implies that the continuum level in P Cyg could vary up to $10 \%$ level, which would affect directly the EW measures at the same level. This also strongly suggests that the variations in EW measures seen in Table 3 are caused by variations in the continuum level and not the emission component itself. 


\section{Summary}

In general, mass-loss rates and stellar wind properties are extremely important characteristics of LBVs. Mass-loss rates for P Cyg have already been estimated through several methods, but none of them rely on spatially resolved $\mathrm{H} \alpha$-emitting inner region. In this observational study we have presented the most comprehensive interferometric data set on P Cyg to date. Our results provide a new and independent source of observational constraints that can be utilized when determining the properties of the wind structure in the context of the predicted $\mathrm{H} \alpha$ intensity distribution on the sky.

We have fitted circularly symmetric uniform disk, Gaussian, and two-component models to the interferometric observations and have shown how the $\mathrm{H} \alpha$-emitting wind structure of P Cyg cannot be represented by a simple one component model. We conclude that the wind structure can be represented fully by a two-component Gaussian model with angular diameters of $\theta_{1}=5.64 \pm 0.21$ mas and $\theta_{2}=1.80 \pm 0.13$ mas. The interferometric signature can also be equally well represented with a two-component model consisting of a uniform disk and a Gaussian intensity distribution. In that case the angular diameters are $\theta_{\mathrm{UD}}=3.06 \pm 0.15$ mas and $\theta_{\mathrm{GD}}=5.46 \pm 0.16$ mas. While we cannot conclude if a double Gaussian model or a uniform-disk plus a Gaussian model better represents the wind structure of $\mathrm{P} \mathrm{Cyg}$, it is clear that both models represent the wind structure better than a single Gaussian or uniform disk model. We conclude that P Cyg's wind structure is complex, possibly containing multiple layers of varying opacities. The data presented in this study might be used to directly constrain wind models of $\mathrm{P}$ Cyg, perhaps allowing for an independent observational constraint on the $\beta$-law.

Based on our spectroscopic and interferometric observations we conclude that $\mathrm{P}$ Cyg is stable at the $10 \%$ level. Based on the photometric observations we also rule out the possibility that large changes in the $\mathrm{H} \alpha \mathrm{EW}$ measure were masked by changes in the continuum level. The lack of variability at a significant level combined with the spatially resolved $\mathrm{H} \alpha$-emitting region that appears to be stable between the observing seasons is suggestive of an extra-quiescent phase of P Cyg between 2005 and 2008.

The Navy Prototype Optical Interferometer is a joint project of the Naval Research Laboratory

and the U.S. Naval Observatory, in cooperation with Lowell Observatory, and is funded by the Office of Naval Research and the Oceanographer of the Navy. We thank Anatoly Miroshnichenko and Glen Williams for valuable suggestions on how to improve the modeling section of this work. We are very grateful for the very detailed and constructive comments and suggestions we have received from the anonymous referee. We would also like to thank the Lowell Observatory for the generous telescope time allocation on the John S. Hall Telescope and Nevena Markova for providing 
us with electronic files of the published data. We acknowledge with thanks the variable star observations of the AAVSO Photoelectric Photometry program contributed by observers worldwide and used in this research. A.B. and C.T. would like to thank Central Michigan University for the financial support for this project. C.T. acknowledges, with thanks, grant support from Research Corporation for Science Advancement. This research has made use of NASA's Astrophysics Data System Bibliographic Services, and the SIMBAD database, operated at CDS, Strasbourg, France.

Facilities: NPOI, Hall (Solar Stellar Spectrograph) 


\section{REFERENCES}

Abbott, D. C., Bieging, J. H., Churchwell, E., \& Cassinelli, J. P. 1980, ApJ, 238, 196

Armstrong, J. T., et al. 1998, ApJ, 496, 550

Barlow, M. J., Drew, J. E., Meaburn, J., \& Massey, R. M. 1994, MNRAS, 268, L29

Benson, J. A., et al. 1997, AJ, 114, 1221

Castor, J. I., \& Lamers, H. J. G. L. M. 1979, ApJS, 39, 481

Chesneau, O., et al. 2000, A\&AS, 144, 523

Crowther, P. A. 2007, ARA\&A, 45, 177

de Groot, M. 1969, Bull. Astron. Inst. Netherlands, 20, 225

de Groot, M. J. H., \& Lamers, H. J. G. L. M. 1992, Nature, 355, 422

Hall, J. C., \& Lockwood, G. W. 1995, ApJ, 438, 404

Hofmann, K.-H., Balega, Y., Ikhsanov, N. R., Miroshnichenko, A. S., \& Weigelt, G. 2002, A\&A, 395,891

Hummel, C. A., et al. 2003, AJ, 125, 2630

Humphreys, R. M., \& Davidson, K. 1994, PASP, 106, 1025

Lamers, H. J. G. L. M., de Groot, M., \& Cassatella, A. 1983, A\&A, 128, 299

Lamers, H. J. G. L. M., Korevaar, P., \& Cassatella, A. 1985, A\&A, 149, 29

Lamers, H. J. G. L. M., et al. 1996, A\&A, 315, L229

Lamers, H. J. G. L. M., \& Cassinelli, J. P. 1999, Introduction to Stellar Winds (Cambridge: Cambridge University Press)

Langer, N., Hamann, W.-R., Lennon, M., Najarro, F., Pauldrach, A. W. A., \& Puls, J. 1994, A\&A, 290,819

Leitherer, C., \& Zickgraf, F.-J. 1987, A\&A, 174, 103

Leitherer, C. 1988, ApJ, 326, 356

Markova, N., Morrison, N., Kolka, I., \& Markov, H. 2001a, A\&A, 376, 898

Markova, N., Scuderi, S., de Groot, M., Markov, H., \& Panagia, N. 2001b, A\&A, 366, 935

Najarro, F., Hillier, D. J., \& Stahl, O. 1997, A\&A, 326, 1117 
Pauldrach, A. W. A., \& Puls, J. 1990, A\&A, 237, 409

Preibisch, T., Balega, Y. Y., Schertl, D., \& Weigelt, G. 2003, A\&A, 412, 735

Scuderi, S., Bonanno, G., Spadaro, D., Panagia, N., Lamers, H. J. G. L. M., \& de Koter, A. 1994, ApJ, 437, 465

Skinner, C. J., Exter, K. M., Barlow, M. J., Davis, R. J., \& Bode, M. F. 1997, MNRAS, 288, L7

Skinner, C. J., Becker, R. H., White, R. L., Exter, K. M., Barlow, M. J., \& Davis, R. J. 1998, MNRAS, 296, 669

Stahl, O., Wolf, B., Gaeng, T., Kaufer, A., Mandel, H., Szeifert, T., \& Zhao, F. 1994, A\&AS, 107, 1

Tycner, C., et al. 2003, AJ, 125, 3378

Tycner, C., et al. 2006, AJ, 131, 2710

Tycner, C., Jones, C. E., Sigut, T. A. A., Schmitt, H. R., Benson, J. A., Hutter, D. J., \& Zavala, R. T. 2008, ApJ, 689, 461

Vakili, F., Mourard, D., Bonneau, D., Morand, F., \& Stee, P. 1997, A\&A, 323, 183

van Blerkom, D. 1978, ApJ, 221, 186 


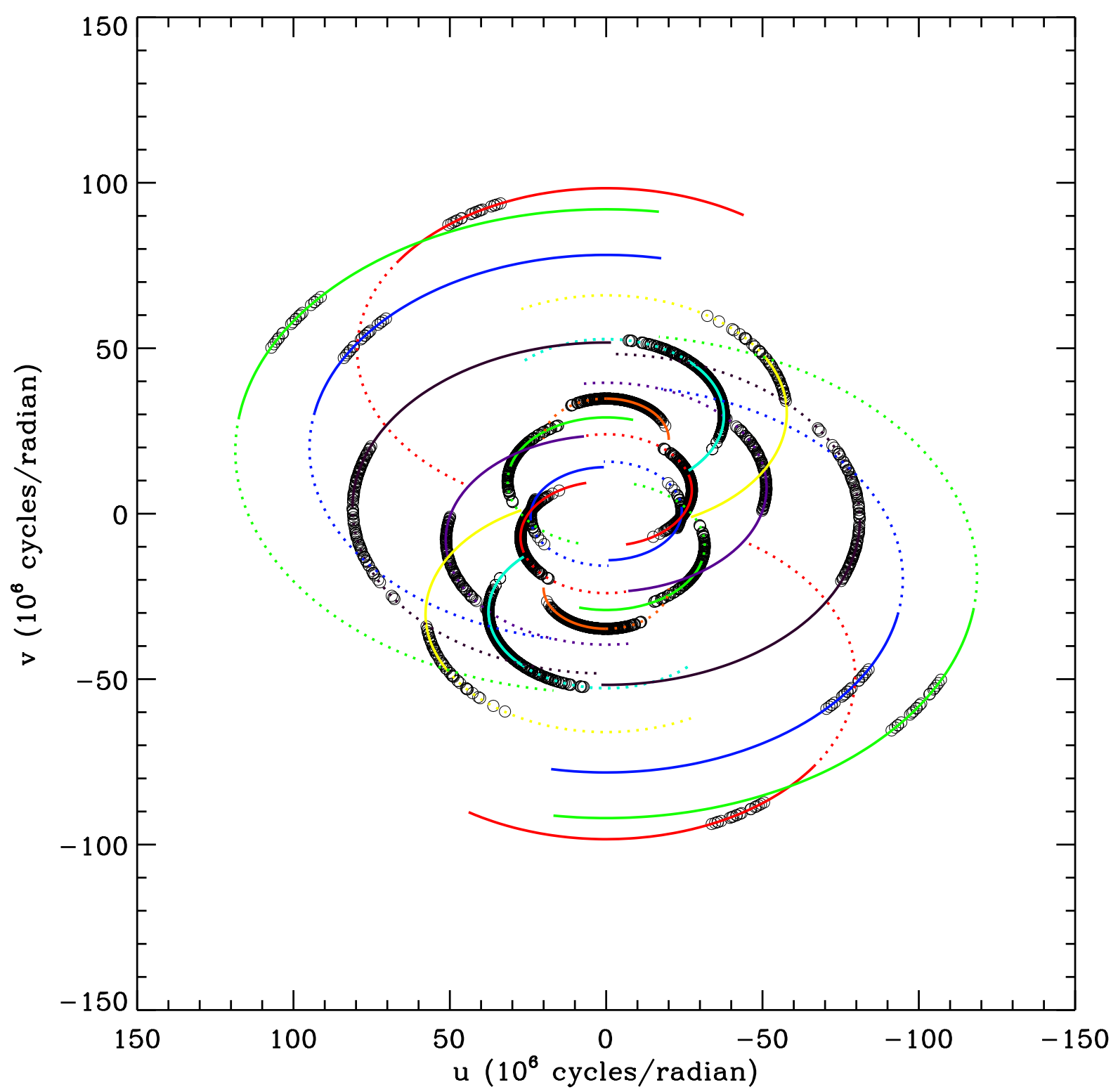

Fig. 1.- Sampling of the $(u, v)$-plane in the $\mathrm{H} \alpha$ channel for $\mathrm{P}$ Cyg on multiple baselines ranging in length from 15.8 to $79.3 \mathrm{~m}$ obtained on thirty-seven nights of observation in 2005, 2007 and 2008 (open circles). Possible coverage at each baseline is also shown from $6 \mathrm{hr}$ east of meridian (dotted line) to $6 \mathrm{hr}$ west of meridian (solid line). 


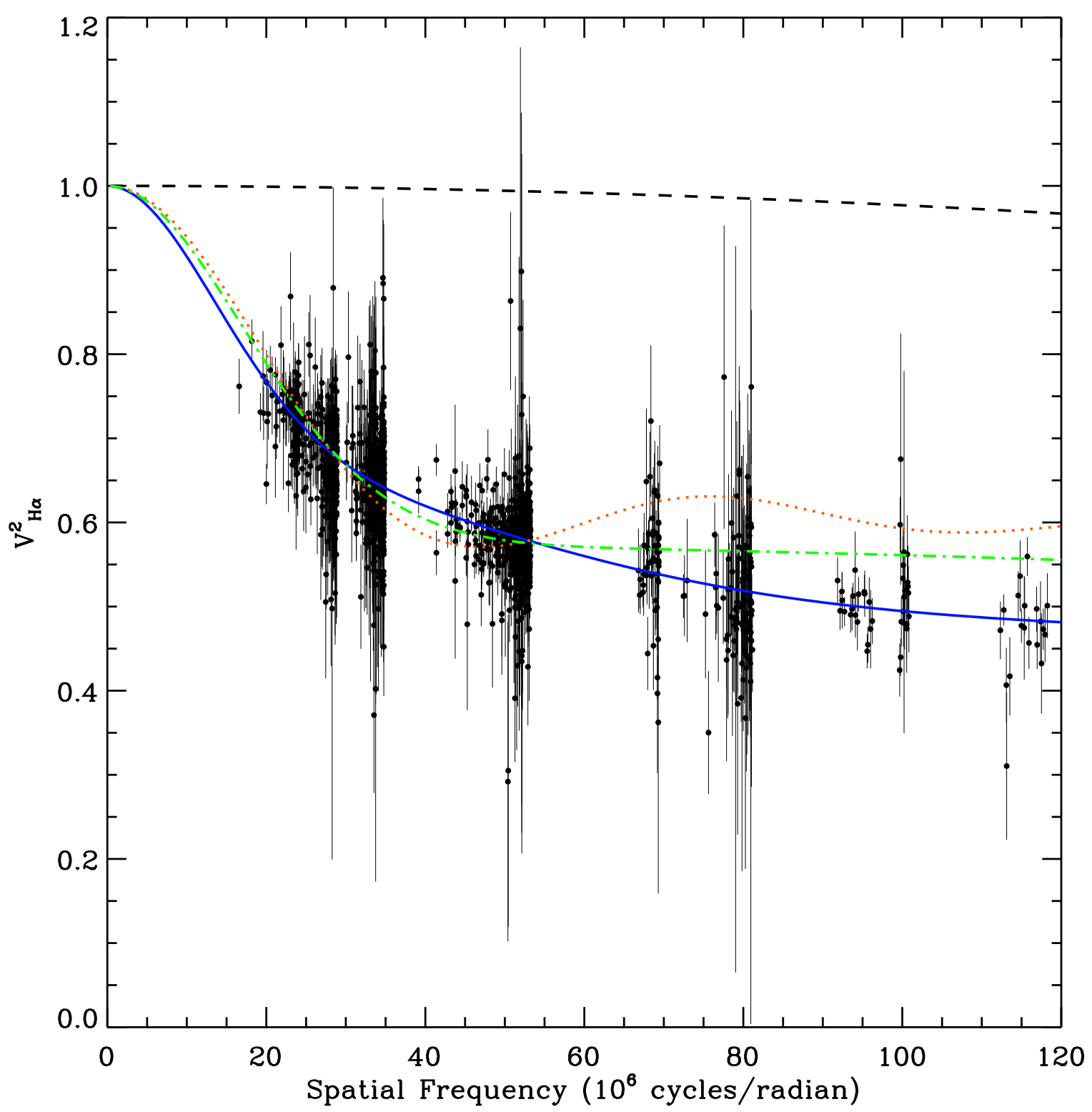

Fig. 2.- The calibrated squared visibilities from the $\mathrm{H} \alpha$ channel of $\mathrm{P}$ Cyg obtained on 11 unique baselines from 37 nights of observation in 2005, 2007 and 2008. A signature of a central star represented by a UD model with angular diameter of 0.2 mas is shown (dashed-line) along with three models representing the $\mathrm{H} \alpha$-emitting envelope. The envelope component is modeled with a uniform disk (dotted-line), a single component Gaussian (dash-dotted-line), and a two-component Gaussian model (solid-line). 

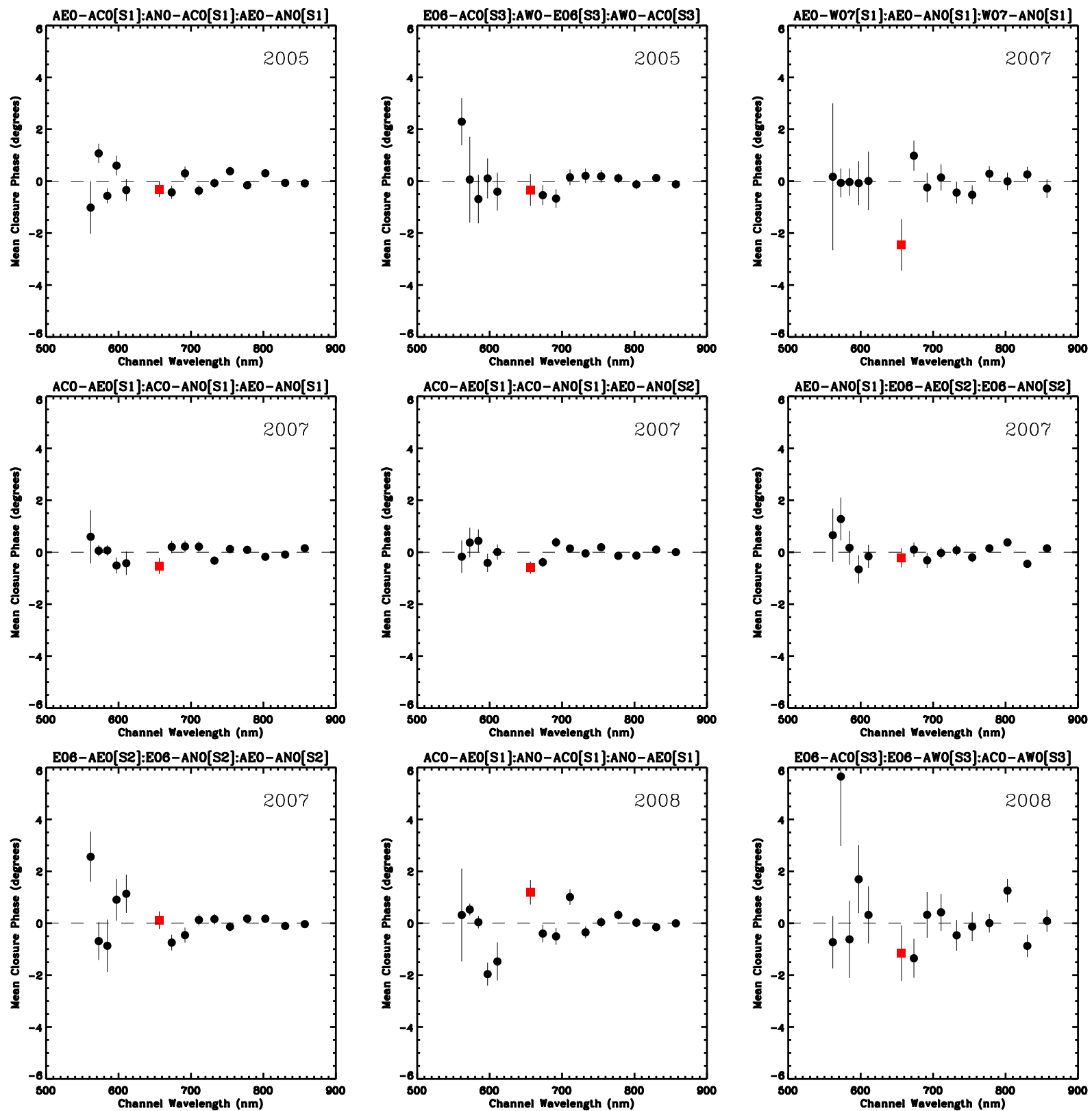

Fig. 3.- Weighted mean closure phases (with a quadratic trend removed from the continuum channels) obtained at each possible baseline triangle obtained in 2005, 2007 and 2008 observing seasons. The signal from the channel containing the $\mathrm{H} \alpha$ emission is marked with red squares. The closure phases containing the $\mathrm{AC}-\mathrm{AE}-\mathrm{AN}$ and $\mathrm{E} 6-\mathrm{AE}-\mathrm{AN}$ stations in the 2007 season are plotted in two panels since one of the baselines (AE-AN) was observed at two independent output beams from the beamcombiner (indicated in the square brackets with a S1 or S2 designator), thereby allowing calculation of two closure phase quantities. The error bars are based on the uncertainty of the weighted average. 


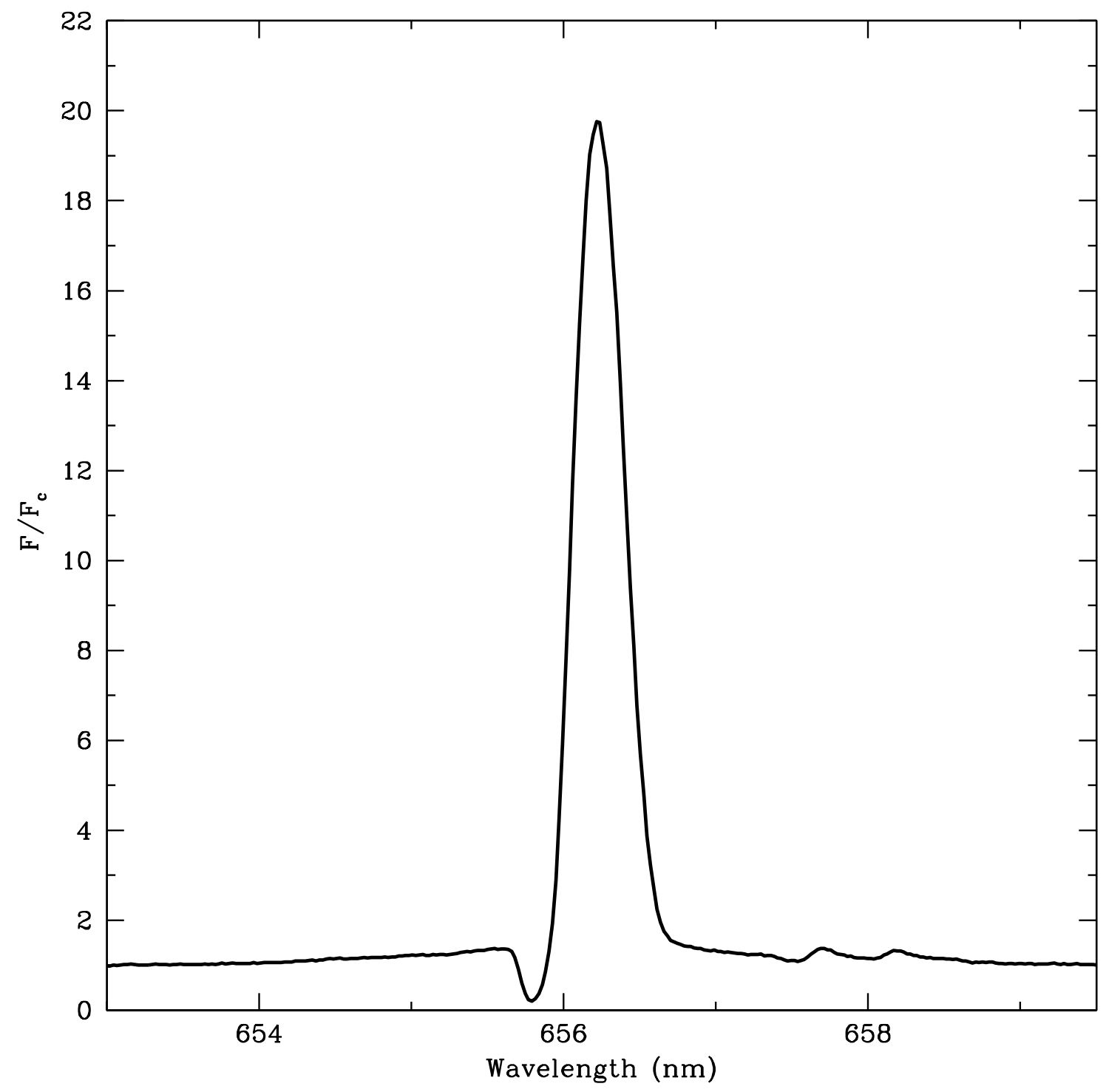

Fig. 4.- A representative $\mathrm{H} \alpha$ line profile of $\mathrm{P}$ Cyg obtained on 2007 June 22. The equivalent width of the emission line is $-80.0 \AA$. 

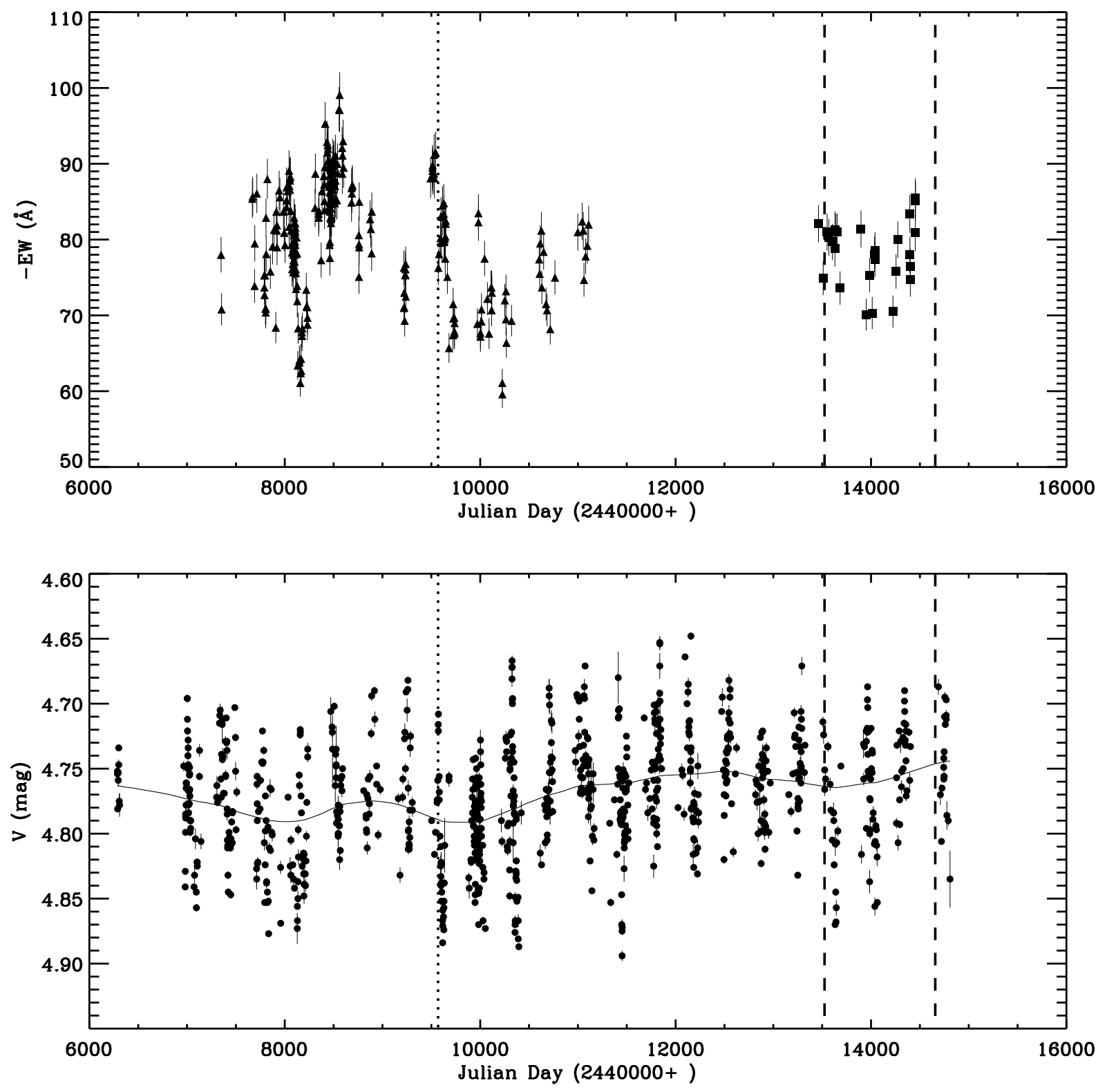

Fig. 5.- Upper panel: P Cyg H $\alpha$ equivalent widths from 1988 Jul to 2007 Dec with data from Markova et al. (2001a) (triangles) and this study (squares) shown. The vertical lines mark the periods when interferometric observations were obtained at the NPOI (dashed lines), and the single night of interferometric observation of $\mathrm{P}$ Cyg reported by Vakili et al. (1997) (dotted line). Lower panel: The AAVSO Johnson V-band photoelectric light curve of P Cyg for the period 2005-2009, obtained based on measurements relative to the comparison star HD $188892(V=4.936, B-V=-0.086)$. Only data where the measured check star magnitude (HD 193369; $V=5.573$ ) lies within \pm 0.04 mag of the known value are plotted. Typical photometric errors per point are 5-10 milli-magnitudes. The smoothed moving average (with a $1000 \mathrm{~d}$ window) is also shown (solid line) to guide the eye for possible long-term variability. 


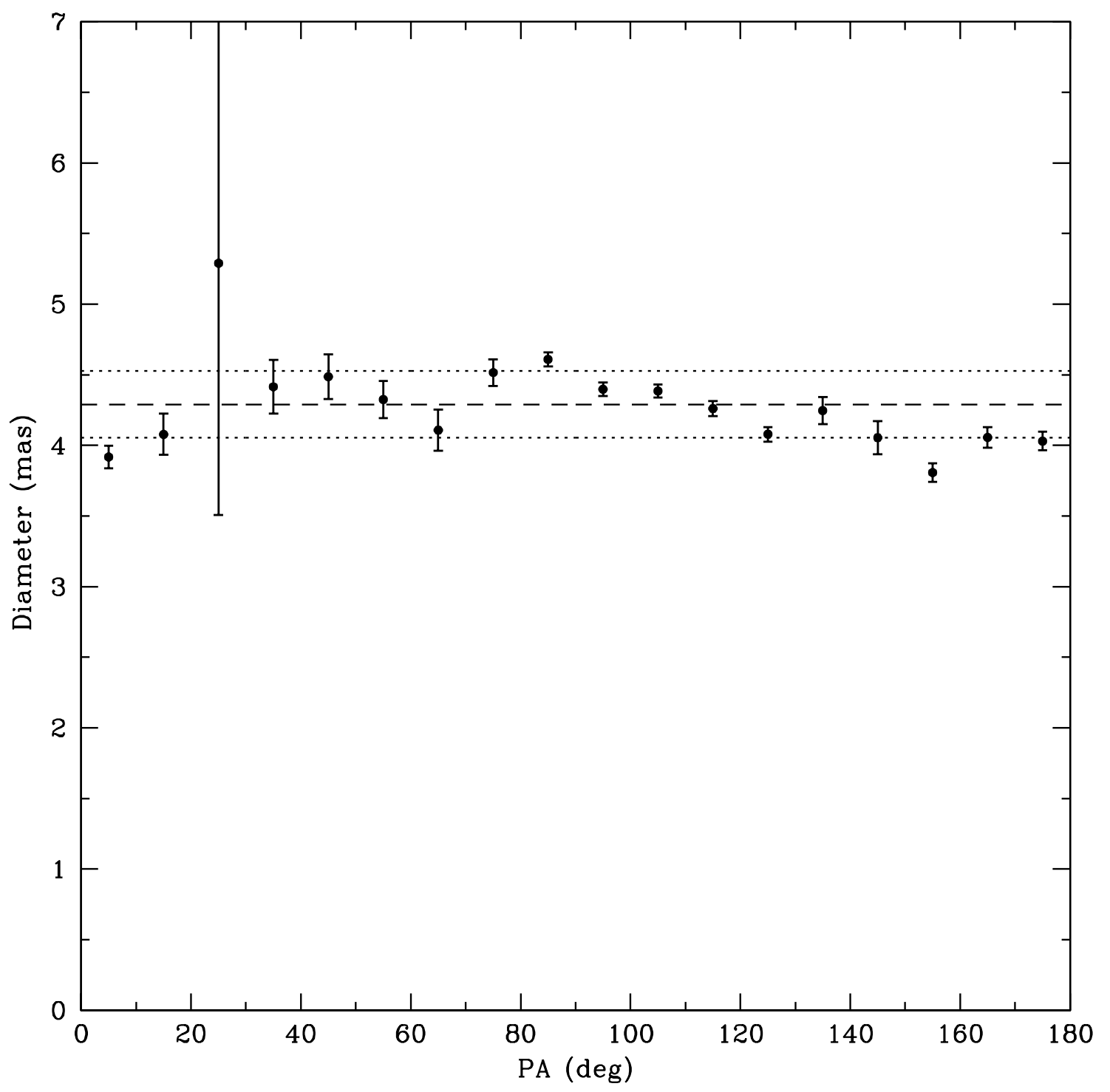

Fig. 6.- Diameter of a Gaussian model fitted to subsets of squared visibility data divided based on ranges of $\mathrm{PA}$ on the sky. Each point represents a $10^{\circ}$ range in $\mathrm{PA}$. The mean diameter of $4.29 \pm 0.03$ mas based on a model fit to all data (dashed line) and $5.5 \%$ variation around that value (dotted lines) are also shown. 
Table 1: NPOI Observing Log of P Cyg

\begin{tabular}{|c|c|}
\hline UT Date & $\begin{array}{c}\# \text { of } \mathrm{H} \alpha \text { squared } \\
\text { visibilities }\end{array}$ \\
\hline 2005 Jun 2 & 48 \\
\hline 2005 Jun 3 & 19 \\
\hline 2005 Jun 4 & 48 \\
\hline 2005 Jun 6 & 18 \\
\hline 2005 Jun 8 & 20 \\
\hline 2005 Jun 9 & 84 \\
\hline 2005 Jun 11 & 19 \\
\hline 2005 Jun 13 & 50 \\
\hline 2005 Jun $16 \ldots \ldots \ldots \ldots$ & 90 \\
\hline 2007 Jul 1 & 20 \\
\hline 2007 Jul 2 & 20 \\
\hline $2007 \mathrm{Jul} 4$ & 20 \\
\hline 2007 Jul 12 & 15 \\
\hline 2007 Aug 8 & 24 \\
\hline 2007 Aug 9 & 16 \\
\hline 2007 Aug 10 & 66 \\
\hline 2007 Aug 11 . & 56 \\
\hline 2007 Aug 12 ........... & 28 \\
\hline 2007 Aug 18 ............ & 72 \\
\hline 2007 Aug 19 ........... & 31 \\
\hline 2007 Aug 20 ............ & 82 \\
\hline 2007 Aug 21 ............ & 33 \\
\hline 2007 Aug 22 ............ & 92 \\
\hline 2007 Aug 23 ............ & 17 \\
\hline 2007 Aug $24 \ldots \ldots$. & 12 \\
\hline 2007 Aug $25 \quad \ldots \ldots \ldots \ldots$ & 41 \\
\hline 2007 Aug 27 ............ & 12 \\
\hline 2007 Aug $29 \ldots \ldots \ldots \ldots$ & 12 \\
\hline 2007 Sep $5 \ldots \ldots \ldots \ldots \ldots$ & 36 \\
\hline 2008 Jun $27 \ldots \ldots \ldots \ldots \ldots$ & 16 \\
\hline 2008 Jun $28 \ldots \ldots \ldots \ldots \ldots$ & 40 \\
\hline 2008 Jun $30 \ldots \ldots \ldots \ldots \ldots$ & 36 \\
\hline 2008 Jul $2 \ldots \ldots$. & 28 \\
\hline $2008 \mathrm{Jul} 3$ & 60 \\
\hline 2008 Jul 7 & 64 \\
\hline 2008 Jul 8 & 89 \\
\hline 2008 Jul 9 & 100 \\
\hline Total ..... & 1534 \\
\hline
\end{tabular}


Table 2: Calibrated $\mathrm{H} \alpha$ Squared Visibilities of P Cyg

\begin{tabular}{crrcc}
\hline \hline $\begin{array}{c}\text { Julian Date } \\
(\mathrm{JD}-2,450,000)\end{array}$ & $\begin{array}{r}\text { Spatial Frequency } u \\
\left(10^{6} \text { cycles/radian }\right)\end{array}$ & $\begin{array}{r}\text { Spatial Frequency } v \\
\left(10^{6} \text { cycles/radian }\right)\end{array}$ & $V_{\mathrm{H} \alpha}^{2}$ & Baseline \\
\hline 3523.863 & -18.364 & 19.654 & $0.666 \pm 0.028$ & AC-AE \\
3523.863 & -11.184 & -32.672 & $0.623 \pm 0.040$ & AC-AN \\
3523.863 & -7.180 & 52.326 & $0.615 \pm 0.017$ & AE-AN \\
3523.863 & -37.854 & 29.298 & $0.567 \pm 0.023$ & AC-E6 \\
3523.863 & 67.722 & -25.810 & $0.512 \pm 0.026$ & AW-E6 \\
3523.863 & 29.868 & 3.488 & $0.671 \pm 0.045$ & AC-AW \\
3523.891 & -21.636 & 17.482 & $0.705 \pm 0.023$ & AC-AE \\
3523.891 & -8.106 & -33.720 & $0.633 \pm 0.039$ & AC-AN \\
3523.891 & -13.530 & 51.202 & $0.515 \pm 0.023$ & AE-AN \\
3523.891 & -43.328 & 24.890 & $0.526 \pm 0.025$ & AC-E6 \\
3523.891 & 74.493 & -18.088 & $0.501 \pm 0.033$ & AW-E6 \\
\hline
\end{tabular}

Note. - Table 2 is published in its entirety in the electronic edition of the Astronomical Journal. 
Table 3: Spectroscopic Variability for P Cyg

\begin{tabular}{lcccc}
\hline \hline UT Date & - EW $(\AA)$ & \% Change & $\frac{F_{\max }}{F_{c}}$ & $\%$ Change $^{\dagger}$ \\
\hline 2005 Apr 1 & 82.1 & 3.5 & 20.8 & 6.7 \\
2005 May 19 & 74.9 & 5.7 & 18.7 & 4.0 \\
2005 Jun 28 & 81.0 & 2.0 & 20.8 & 6.7 \\
2005 Jul 16 & 80.3 & 1.1 & 20.8 & 6.9 \\
2005 Aug 22 & 79.7 & 0.5 & 19.7 & 1.1 \\
2005 Sep 16 & 78.8 & 0.8 & 19.1 & 2.0 \\
2005 Sep 17 & 81.3 & 2.4 & 19.6 & 0.6 \\
2005 Sep 27 & 81.0 & 2.1 & 19.5 & 0.1 \\
2005 Oct 11 & 81.0 & 2.1 & 18.9 & 3.1 \\
2005 Nov 8 & 73.6 & 7.3 & 17.0 & 12.9 \\
\hline 2005 Mean & $79.4 \pm 0.9$ & & $19.5 \pm 0.4$ & \\
\hline 2006 Jun 10 & 81.4 & 7.3 & 19.7 & 7.3 \\
2006 Aug 3 & 70.1 & 7.7 & 16.6 & 9.4 \\
2006 Sep 6 & 75.3 & 0.8 & 17.9 & 2.7 \\
2006 Oct 3 & 70.3 & 7.3 & 17.2 & 6.2 \\
2006 Oct 28 & 78.1 & 2.9 & 19.0 & 3.3 \\
2006 Oct 31 & 77.4 & 2.0 & 19.0 & 3.4 \\
2006 Nov 1 & 78.6 & 3.5 & 19.2 & 4.4 \\
\hline 2006 Mean & $75.9 \pm 1.6$ & & $18.4 \pm 0.4$ & \\
\hline 2007 May 3 & 70.5 & 10.8 & 17.3 & 17.7 \\
2007 Jun 4 & 75.8 & 4.1 & 18.7 & 11.1 \\
2007 Jun 22 & 80.0 & 1.2 & 19.7 & 6.4 \\
2007 Oct 23 & 83.4 & 5.5 & 22.5 & 6.8 \\
2007 Oct 24 & 78.0 & 1.3 & 21.1 & 0.3 \\
2007 Oct 27 & 76.5 & 3.2 & 20.9 & 0.6 \\
2007 Oct 28 & 74.7 & 5.6 & 20.2 & 3.9 \\
2007 Dec 18 & 80.9 & 2.4 & 22.6 & 7.5 \\
2007 Dec 19 & 85.5 & 8.2 & 23.7 & 12.7 \\
2007 Dec 20 & 85.2 & 7.8 & 23.7 & 12.5 \\
\hline 2007 Mean & $79.0 \pm 1.6$ & & $21.0 \pm 0.7$ & \\
\hline
\end{tabular}

$\dagger$ Changes calculated with respect to yearly mean EW and peak strength. 
Table 4: Best-Fit Model Parameters for P Cyg

\begin{tabular}{|c|c|c|c|c|c|c|}
\hline Model & Season & Diameter (mas) & $2^{\text {nd }}$ Diameter (mas) & $k_{1}$ or $l_{1}$ & $c_{\mathrm{p}}$ & $\overline{\chi_{\nu}^{2}}$ \\
\hline Uniform Disk $^{\dagger} \ldots$ & 2005 & $9.41 \pm 2.50$ & $\ldots$ & $\ldots$ & $0.84 \pm 0.03$ & \\
\hline Uniform Disk ... & 2005 & $6.74 \pm 0.05$ & $\ldots$ & $\ldots$ & $0.78 \pm 0.01$ & 2.6 \\
\hline Gaussian .... & 2005 & $3.97 \pm 0.05$ & $\ldots$ & $\ldots$ & $0.75 \pm 0.01$ & 1.5 \\
\hline Double-Gaussian ....... & 2005 & $8.7 \pm 5.3$ & $2.08 \pm 0.15$ & $0.41 \pm 0.03$ & $0.70 \pm 0.01$ & 1.2 \\
\hline Uniform Disk $^{\dagger} \ldots \ldots \ldots$ & 2007 & $10.17 \pm 0.32$ & $\ldots$ & $\ldots$ & $0.84 \pm 0.01$ & \\
\hline Uniform Disk ... & 2007 & $7.37 \pm 0.03$ & $\ldots$ & $\ldots$ & $0.79 \pm 0.01$ & 3.7 \\
\hline Gaussian .... & 2007 & $4.37 \pm 0.03$ & $\ldots$ & $\ldots$ & $0.76 \pm 0.01$ & 2.4 \\
\hline Double-Gaussian & 2007 & $5.33 \pm 0.15$ & $1.53 \pm 0.15$ & $0.60 \pm 0.02$ & $0.70 \pm 0.01$ & 1.7 \\
\hline Uniform Disk $^{\dagger} \ldots$ & 2008 & $8.40 \pm 1.50$ & $\cdots$ & $\cdots$ & $0.79 \pm 0.04$ & \\
\hline Uniform Disk . & 2008 & $7.06 \pm 0.06$ & $\ldots$ & $\ldots$ & $0.78 \pm 0.01$ & 1.5 \\
\hline Gaussian ....... & 2008 & $4.37 \pm 0.07$ & $\ldots$ & $\ldots$ & $0.75 \pm 0.01$ & 1.3 \\
\hline Double-Gaussian $\ddagger$ & 2008 & $10 \pm 9$ & $2.17 \pm 0.07$ & $0.50 \pm 0.02$ & 0.70 & 1.1 \\
\hline Uniform Disk $^{\dagger} \ldots$ & All & $9.83 \pm 0.26$ & $\ldots$ & $\ldots$ & $0.84 \pm 0.01$ & \\
\hline Uniform Disk . & All & $7.21 \pm 0.03$ & $\ldots$ & $\ldots$ & $0.79 \pm 0.01$ & 2.8 \\
\hline Gaussian ....... & All & $4.29 \pm 0.03$ & $\ldots$ & $\ldots$ & $0.76 \pm 0.01$ & 1.9 \\
\hline Double-Gaussian ....... & All & $5.64 \pm 0.17$ & $1.80 \pm 0.11$ & $0.57 \pm 0.02$ & $0.70 \pm 0.01$ & 1.5 \\
\hline Uniform Disk + Gaussian & All & $3.06 \pm 0.15$ & $5.46 \pm 0.16$ & $0.36 \pm 0.02$ & $0.72 \pm 0.01$ & 1.5 \\
\hline
\end{tabular}

$\dagger$ Models fitted to data for baselines up to $18.9 \mathrm{~m}$.

$\ddagger$ There were insufficient data at the longest baselines to fit for four model parameters and instead a fit was obtained with $c_{\mathrm{p}}$ fixed at 0.70 . 
Table 5: Single Night Spectroscopic Variability for P Cyg

\begin{tabular}{lcccc}
\hline \hline UT Date & - EW $(\AA)$ & \% Change & $\frac{F_{\max }}{F_{c}}$ & \% Change $^{\dagger}$ \\
\hline 2007 Dec 20 & 85.2 & 0.1 & 23.7 & 0.0 \\
2007 Dec 20 & 85.9 & 0.9 & 23.8 & 0.7 \\
2007 Dec 20 & 84.4 & 0.9 & 23.5 & 0.7 \\
2007 Dec 20 & 85.6 & 0.5 & 23.8 & 0.4 \\
2007 Dec 20 & 85.2 & 0.1 & 23.7 & 0.0 \\
2007 Dec 20 & 85.8 & 0.7 & 23.8 & 0.6 \\
2007 Dec 20 & 86.0 & 1.0 & 23.8 & 0.6 \\
2007 Dec 20 & 83.4 & 2.1 & 23.4 & 1.2 \\
2007 Dec 20 & 85.0 & 0.2 & 23.6 & 0.2 \\
2007 Dec 20 & 85.2 & 0.0 & 23.7 & 0.0 \\
2007 Dec 20 & 83.4 & 2.1 & 23.4 & 1.2 \\
2007 Dec 20 & 84.7 & 0.6 & 23.6 & 0.4 \\
2007 Dec 20 & 85.3 & 0.1 & 23.6 & 0.1 \\
2007 Dec 20 & 85.5 & 0.4 & 23.7 & 0.3 \\
2007 Dec 20 & 85.4 & 0.2 & 23.7 & 0.1 \\
2007 Dec 20 & 85.7 & 0.6 & 23.8 & 0.5 \\
2007 Dec 20 & 86.1 & 1.1 & 23.8 & 0.6 \\
2007 Dec 20 & 85.5 & 0.4 & 23.7 & 0.3 \\
2007 Dec 20 & 85.1 & 0.1 & 23.6 & 0.3 \\
\hline 2007 Dec 20 Mean & $85.2 \pm 0.2$ & & $23.67 \pm 0.03$ & \\
\hline
\end{tabular}

${ }^{\dagger}$ Changes calculated with respect to nightly mean. All spectra were obtained with $180 \mathrm{~s}$ of integration. 